phenotypic diversity in GSC subpopulations, together suggesting that not all GSCs are equivalent. These observations should be considered when studying GSCs in the laboratory, with implications for the development of treatments that target GSCs and prevent tumor recurrence in patients.

\section{STEM-18. DEVELOPMENT OF A NOVEL GENE THERAPY APPROACH TARGETING GLIOBLASTOMA FOLLOWING AI-DIRECTED IDENTIFICATION OF A MASTER REGULATORY GENE NETWORK \\ Son $\mathrm{Le}^{1}$, Bo Ma ${ }^{1}$, Sergei Zolotukhin ${ }^{1}$, Darin Falk ${ }^{2}$, and David Tran ${ }^{1}$; ${ }^{1}$ University of Florida, Gainesville, FL, USA, ${ }^{2}$ Lacerta Therapeutics, Allachua, FL, USA}

BACKGROUND: Profound heterogeneity has severely hampered therapeutic advancements in glioblastoma (GBM). Remarkably, GBM exhibits broad clinical and histopathologic overlaps suggesting the presence of a common regulatory state. The GBM state embodies restructuring of the master regulatory gene network (MRGN) forced by founding mutations and perpetuated in subclones of GBM stem-like cells (GSC). Successful targeting and altering of the MRGN promise to circumvent the heterogeneity. METHODS: To decipher the common MRGN in GSC, we applied a robust AI suite, NETZEN, that integrates deep neural networks with gene network-based ranking to the reference GBM network generated using TCGA's entire GBM RNAseq dataset and a dataset containing > 30 diverse GSC and paired GBM differentiated cell lines, 6 normal astrocyte and 3 normal neuronal precursor cell lines. To develop a gene therapy against the MRGN, we performed a screen of a rAAV capsid library using GBM patient-derived orthotopic xenografts (PDX) to identify capsid variants with specific tropism for GBM cells to deliver shRNA-based cassettes. RESULTS: We discovered a putative MRGN in GSC, anchored by developmentally restricted master regulators (MR). To confirm its critical role, we deconstructed it using shRNA in a panel of PDX and uniformly observed attenuated tumor growth and mortality compared to controls $(\mathrm{p}<0.05$ in all lines). More notably, when normal astrocytes were forced to reconstruct the MRGN, transformation into GSC-like cells occurred, as measured by single-cell analysis, sphere formation in vitro, and most importantly, development of lethal infiltrating brain tumors in $15 / 15$ mice. Finally, selected novel AAV capsids with 10-40-fold higher specificity for GBM cells were utilized in a shRNA-based AAV platform to target key MR in the validated MRGN. CONCLUSIONS: The GBM state is established by a developmental subnetwork permitting a first-of-its-kind, heterogeneity-agnostic AAV-based treatment approach. This AI-directed R\&D program can be expanded to target other cancers.

\section{STEM-19. THE ISOLATION AND IDENTIFICATION OF GLIOMA STEM CELLS AND MESENCHYMAL STEM CELLS FROM HUMAN GLIOMA SPECIMENS}

Yan-jiao Yu, Wang Jing, and Zhong-ping Chen; Sun Yat-sen University Cancer Center, Guangzhou, China (People's Republic)

INTRODUCTION: Glioma is the primary malignancy with the highest incidence in central nervous system. Studies have shown that glioma stem cell (GSC) is the important origin for recurrence and closely associated with tumor microenvironment. Glioma-associated human mesenchymal stem cells (GA-hMSCs), as an important part of the tumor microenvironment might promote proliferation and invasion of GSC. We try to isolate GSCs and GA-hMSCs through glioma primary culture, and investigate the relationship between GSC and GA-hMSCs, as well as their roles in the recurrences of glioma. MATERIALS AND METHODS: Five surgical specimens from patients with human high-grade glioma were collected for primary culture. The surgical specimens were cut up and digested with Dispase II (collagenase), terminated by adding plenty of DNase II. Cell suspension was collected and resuspended in conditional medium for MSC or GSC. After being cultured for several passages, GSCs were identified by immunofluorescence, and GA-hMSCs were identified by flow cytometry and in vitro differentiation assays. RESULTS: Cell cultures were observed with an in verted phase-contrast microscope after 24 hours. Adherent GA-hMSCs were collected and the supernatant was discarded with non-adherent cells. GSC was cultured as neurospheres. The single-cell suspension was prepared from spheres of GSC to identify their surface expression profile by immunofluorescence assays after several passages. The results confirmed that GSCs positively expressed CD133, Nestin and SOX2, but did not express GFAP and Tublin III. GA-hMSCs positively expressed CD105, CD73, CD90 and negatively expressed CD45 and CD34. GA-hMSCs could differentiate into adipocytes, chondrocytes and osteocytes. CONCLUSION: We isolated and identified GSCs and GA-hMSCs from glioma primary culture. Our future work will focus on the relationship between the two types of cells and their roles in the process of tumor recurrence based on the successful isolation of GSC and GA-hMSCs. Keywords: Glioma; Mesenchymal stem cells; Glioma stem cells; Recurrence
STEM-20. A CANCER STEM CELL-SELECTIVE APOPTOSISINDUCING SMALL MOLECULE FOR THE TREATMENT OF GBM Stephen Skirboll ${ }^{1}$, Natasha Lucki ${ }^{2}$, Genaro Villa ${ }^{3}$, Naja Vergani ${ }^{4}$, Michael Bollong ${ }^{5}$, Brittney Beyer ${ }^{5}$, Jae Wook Lee ${ }^{6}$, Justin Anglin 7 , Stephan Spangenberg ${ }^{6}$, Emily Chin $^{6}$, Amandeep Sharma ${ }^{6}$, Kevin Johnson ${ }^{8}$, Phillipp Sander ${ }^{6}$, Perry Gordon ${ }^{8}$, Heiko Wurdak ${ }^{9}$, Peter Schultz ${ }^{6}$, Paul Mischel ${ }^{10}$, and Luke Lairson ${ }^{6} ;{ }^{1}$ Stanford University School of Medicine, Stanford, CA, USA, ${ }^{2}$ Thermo Fisher Scientific, Waltham, MA, USA, ${ }^{3}$ Brigham and Women's Hospital, Boston, MA, USA, ${ }^{4}$ California Institute for Biomedical Research, La Jolla, CA, USA, ${ }^{5}$ Scripps Research Institute, La Jolla, CA, USA, ${ }^{6}$ The Scripps Research Institute, La Jolla, CA, USA, ${ }^{7}$ Baylor College of Medicine, Houston, TX, USA, ${ }^{8}$ The Genomics Institute of the Novartis Research Foundation, San Diego, CA, USA, ${ }^{9}$ University of Leeds, Leeds, United Kingdom, ${ }^{10}$ Ludwig Institute of Cancer Research, UCSD, San Diego, CA, USA

INTRODUCTION: Glioblastoma multiforme (GBM) is the most aggressive form of primary brain cancer. A subpopulation of multipotent cells termed GBM cancer stem cells (CSCs) play a critical role in tumor initiation and maintenance, drug resistance, and recurrence following surgery. New therapeutic strategies for the treatment of GBM have recently focused on targeting CSCs. Here we have used an unbiased large-scale screening approach to identify drug-like small molecules that induce apoptosis in GBM CSCs in a cell type-selective manner. METHODS: A luciferase-based survival assay of patient-derived GBM CSC lines was established to perform a large-scale screen of $\sim$ one million drug-like small molecules with the goal of identifying novel compounds that are selectively toxic to chemoresistant GBM CSCs. Compounds found to kill GBM CSC lines as compared to control cell types were further characterized. A caspase activation assay was used to evaluate the mechanism of induced cell death. A xenograft animal model using patient-derived GBM CSCs was employed to test the leading candidate for suppression of in vivo tumor formation. RESULTS: We identified a small molecule, termed RIPGBM, from the cell-based chemical screen that induces apoptosis in primary patient-derived GBM CSC cultures. The cell type-dependent selectivity of RIPGBM appears to arise at least in part from redox-dependent formation of a proapoptotic derivative, termed cRIPGBM, in GBM CSCs. cRIPGBM induces caspase 1-dependent apoptosis by binding to receptor-interacting protein kinase 2 (RIPK2) and acting as a molecular switch, which reduces the formation of a prosurvival RIPK2/TAK1 complex and increases the formation of a proapoptotic RIPK2/caspase 1 complex. In an intracranial GBM xenograft mouse model, RIPGBM was found to significantly suppress tumor formation. CONCLUSIONS: Our chemical genetics-based approach has identified a small molecule drug candidate and a potential drug target that selectively targets cancer stem cells and provides an approach for the treatment of GBMs.

\section{STEM-21. CLINICAL RELEVANCE OF CANCER STEM CELL CYTOTOXICITY ASSAY IN GUIDING TREATMENT FOR GLIOBLASTOMA}

Claudio Pier Paolo ${ }^{1}$, Tulika Ranjan $^{2}$, Candace Howard ${ }^{3}$, Alexander $\mathrm{Yu}^{2}$, Linda $\mathrm{Xu}^{2}$, Khaled Aziz ${ }^{2}$, David Jho ${ }^{2}$, Jodi Leonardo ${ }^{2}$, Krista Denning ${ }^{6}$, and Jagan Valluri ${ }^{4} ;{ }^{1}$ University of Mississippi, Jackson, MS, USA, ${ }^{2}$ Allegheny Health Network Cancer Institute, Pittsburgh, PA, USA, ${ }^{3}$ University of Mississippi Medical Center, Jackson, MS, USA, ${ }^{4}$ Marshall University, Huntington, WV, USA

BACKGROUND. The prognosis of glioblastoma (GBM) treated with standard-of-care regimens remains very poor with a median time to recurrence of 7-9 months, and a median survival of 15-18 months. GBMs contain a small, but resilient population of cancer stem cells (CSCs) that contribute to tumor initiation, maintenance, and therapy resistance. For these reasons, its is extremely important to determine the sensitivity of CSCs to chemotherapeutic agents, with the intent of identifying more effective treatment protocols which would then translate into improved clinical survival. METHODS. We have used a novel CLIA and CAP-accredited Cancer Stem Cell Cytotoxicity Assay (ChemoID) to guide treatment for 55 Grade III and Grade IV glioma patients with the most efficacious chemotherapy treatments from a panel of FDA approved drugs or their combinations. Patients were evaluated by MRI scans and response was assessed according to RANO criteria. RESULTS: We report here a prospective clinical investigation using the ChemoID assay to measure the sensitivity and resistance of CSCs and bulk of tumor cells cultured from 55 GBM clinical specimens challenged with several chemotherapy agents, which were also correlated to the clinical response of the treated patients, independently of other biomarkers. The median recurrence time was 15 months for patients with a sensitive ( $>40 \%$ cell kill) CSCs test versus only 6 months for patients with a resistant CSCs test. CONCLUSIONS. The data suggests that GBM patients treated with CSC Cytotoxicity Assay-guided responsive drugs have delayed time to recurrence and the assay has the potential to help tailor chemotherapy choices to improve clinical outcomes. 